\title{
Epidermal Growth Factor Stimulates Rabbit Achilles Tendon Histologically and Biomechanically Healing
}

\author{
I. A. Kocyigit ${ }^{1}$, G. Huri², S. Yürüker ${ }^{3}$, R. Hashemihesar ${ }^{3}$, P. Yilgor Huri', \\ J. Nyland ${ }^{6}$, M. N. Doral ${ }^{2,5}$ \\ 1 Department of Orthopedics and Traumatology, Polatlı Duatepe State Hospital, Polatlı, Ankara, Turkey \\ 2 Department of Orthopedics and Traumatology, School of Medicine, Hacettepe University, Ankara, Turkey \\ 3 Department of Histology and Embryology, School of Medicine, Usak University, Usak, Turkey \\ 4 Department of Biomedical Engineering, Faculty of Engineering, Ankara University, Ankara, Turkey \\ ${ }^{5}$ Department of Orthopedics and Traumatology, School of Medicine, Ufuk University, Ankara, Turkey \\ 6 Department of Orthopaedic Surgery, University of Louisville, Louisville, KY, USA
}

\section{CORRESPONDING AUTHOR: \\ Ismail Aykut Kocyigit \\ Department of Orthopedics and \\ Traumatology \\ Polatlı Duatepe State Hospital \\ Polatlı, Ankara, Turkey \\ E-mail: ismailaykutkocyigit@yahoo.com}

DOI:

10.32098/mltj.04.2020.06

LEVEL OF EVIDENCE: 2B

\begin{abstract}
SUMMARY
Background. Epidermal Growth Factor (EGF) stimulates epidermis cell growth, proliferation and differentiation in skin regeneration. The aim of this study was to pre-clinically investigation of the role of EGF in tendon healing.

Methods. One cm defects were created at the right Achilles tendons of $30 \mathrm{New}$ Zealand White rabbits. Ten rabbits were allocated to one of three groups: Group-1-(Sham) tendon defect with a gap that was splinted with a non-absorbable suture; Group-2-(EGF +) tendon defect with a gap that was splinted with a non-absorbable suture and a $25 \mu \mathrm{g} / \mathrm{kg}$ EGF injection into the defect; Group-3-(Scaffold + EGF) tendon defect was grafted with a biodegradable, porous Polycaprolactone (PCL) scaffold loaded with $25 \mu \mathrm{g} / \mathrm{kg}$ EGF and stabilized with a non-absorbable suture. Animals were sacrificed at 8 weeks post-surgery and Achilles tendon repair and healing status was investigated using histopathologic and biomechanical analysis methods.

Results. Group-2-(EGF +) had greater adipocyte development (moderate) than Group1-(Sham) and Group-3-(Scaffold + EGF). Group-2-(EGF +) and Group-3-(Scaffold + EGF) had greater peripheral nerve development (weak) than Group-1-(Sham). Group-2$(\mathrm{EGF}+)$ had greater vascularization (moderate) than Group-1-(Sham) and Group-3-(Scaffold + EGF). Group-2-(EGF +) had greater collagen Type-III development (moderate) than Group-1-(Sham) and Group-3-(Scaffold + EGF). Group-3-(Scaffold + EGF) had greater collagen Type-I development (moderate) than Group-1-(Sham) and Group2-(EGF +). Groups did not display statistically significant differences for load to failure or elongation at failure. Group-2-(EGF +) and Group-3-(Scaffold + EGF) displayed less stiffness that the control (healthy contralateral Achilles tendon) $(\mathrm{p}<0.05)$, however, experimental groups did not differ ( $\mathrm{p}>0.05$ ).

Conclusions. The application of EGF and scaffold displayed superior histological tendon healing evidence, but there was no significant difference in terms of biomechanics.
\end{abstract}

\section{KEY WORDS}

Achilles tendon; tendon regeneration; epidermal growth factor; scaffold; rabbit.

\section{INTRODUCTION}

The Achilles tendon is the strongest tendon of the ankle joint, and one of the most important biomechanical structures in human gait (1). The Achilles tendon is frequently affected by ankle trauma and it is susceptible to acute or chronic injuries. As a result of these injuries, patients experience significant daily activity and sports performance impairments, functional limitations and disabilities.

Although many studies have been conducted to identify the causes of Achilles tendon rupture, its nature is still not clear- 
ly understood. There is no consensus as to whether Achilles tendon rupture is the result of tendon disease, biomechanical loads, or some combination of both (2). Degenerative changes identified in biopsy specimens obtained during surgical repair suggest that acute Achilles tendon rupture may actually represent acute tears that occur in association with chronic tendon degenerative conditions $(1,3-5)$.

It has been suggested that biologic agents such as growth factors accelerate surgical tendon repair healing and reduce adhesion formation, particularly if they are administered within the first 10 days post-surgery $(6,7)$. Transforming Growth Factor- $\beta$ (TGF- $\beta$ ) for example, has been shown to be particularly active near the proximal Achilles tendon defect, whereas Insulin-like Growth Factor (IGF) and Fibroblast Growth Factor (FGF) are more active throughout the entire defect area. Platelet-Derived Growth Factor (PDGF) and Vascular Endothelial Growth Factor (VEGF) activity was noted throughout the entire defect repair area (7-9).

Although bioactive agents such as Platelet-Rich Plasma (PRP), bone marrow aspiration, Mesenchymal Stem Cells (MSCs) and previously mentioned growth factors are being used as supplements to conventional treatment protocols, Epidermal Growth Factor (EGF) is not currently used for tendon healing. Rather, EGF is routinely used for skin healing, especially among patients with diabetic wounds to facilitate epidermal bridging either by topical infiltration or by intralesional or perilesional injections (10). Injection of EGF in these cases stimulates the growth and proliferation of vascular endothelial cells, keratinocytes and fibroblasts that have an important role in scar tissue formation. Clinical EGF application for subcutaneous wound healing is used worldwide (11). Based on the lack of information regarding the potential role of EGF for tendon healing, the objective of this study was to investigate its efficacy in EGF alone and EGF together with a Polycaprolactone (PCL) scaffold using a rabbit model. The study hypothesis was that use of EGF alone and with a polycaprolactone (PCL) scaffold would display superior Achilles tendon defect healing compared to a sham procedure.

\section{METHODS}

\section{Preparation of the PCL scaffold as the EGF delivery system}

Porous PCL scaffolds were prepared with freeze-drying as previously described (12). Briefly, PCL was dissolved in dichloromethane $(4 \%, 10 \mathrm{~mL})$, poured into petri dishes and lyophilized (Labconco Freezone 6, USA) after freezing at $-20{ }^{\circ} \mathrm{C}$ overnight. Cylindrical porous scaffolds (D: $0.5 \mathrm{~mm}$,
L: $1 \mathrm{~cm}$ ) were cut from the lyophilized foams. EGF (for a final concentration of $25 \mu \mathrm{g} / \mathrm{kg}$ when implanted) was loaded into the scaffolds by adsorption into the scaffold pores. For this, $85 \mu \mathrm{g}$ EGF was suspended in $100 \mu \mathrm{L}$ of $1 \%$, w $/ \mathrm{v}$ alginic acid solution and introduced to both sides of the scaffolds. After air- drying, the scaffolds were dipped into ethanol and then kept in $5 \% \mathrm{w} / \mathrm{v} \mathrm{CaCl}$ for $1 \mathrm{~h}$ to crosslink the alginic acid. Scaffolds were sterilized using ethylene oxide (Steri-Vac gas sterilizer $5 \mathrm{XL}$ ) at $37^{\circ} \mathrm{C}$ for $4 \mathrm{~h} 45 \mathrm{~min}$.

\section{Animals}

Thirty New Zealand White rabbits between 9-12 months old with an average body weight of $3350 \pm 13$ g were included in the study. In this randomized and controlled experimental study, 3 groups of 10 rabbits were created. All procedures were performed after obtaining approval from Animal Experiments Local Ethical Committee (13).

\section{Surgical technique}

A $1 \mathrm{~cm}$ long defect was created in the right Achilles tendon of each rabbit. All surgical procedures were performed by the same surgeon using the same suture material (3.0 Prolene, Ethicon, USA) for tendon repair. No operations were performed on the left Achilles tendons, which constituted the control group.

All animals were administered $20 \mathrm{mg} / \mathrm{kg}$ Cefazolin Sodium I.M. antibiotic prophylaxis and then were anesthetized using $35 \mathrm{mg} / \mathrm{kg}$ ketamine HCL and $5 \mathrm{mg} / \mathrm{kg}$ xylazine. A sufficient level of anesthetic depth was reached after corneal reflexes disappeared.

The surgery site was dyed using an antiseptic solution of $10 \%$ Batticon ${ }^{\circledR}$ and the rabbits were positioned in prone. After surgical site sterilization, the Achilles tendon was palpated and an approximately $4 \mathrm{~cm}$ long skin incision was made starting from the ankle posterior and extending proximally along the medial side of the Achilles tendon. Subcutaneous tissue was sharply dissected, and the Achilles tendon was reached. While the rabbit hind sole parallel to the ground, surgical pen and ruler were used to demarcate the region between $1.5 \mathrm{~cm}$ and $2.5 \mathrm{~cm}$ from the Achilles tendon calcaneal insertion (figure 1 a).

The marked tendon regions on the right Achilles tendons of all rabbits were excised to create a $1 \mathrm{~cm}$ long Achilles tendon defect (figure $1 \mathbf{b}$ ). After defect creation, surgical repair was performed using non-absorbable suture and the same suture method (modified Kessler technique) at the right Achilles tendon for each group of rabbits (figure $\mathbf{1} \mathbf{c}$ ). A different interventional procedure was then performed in each of the 3 experimental groups. 

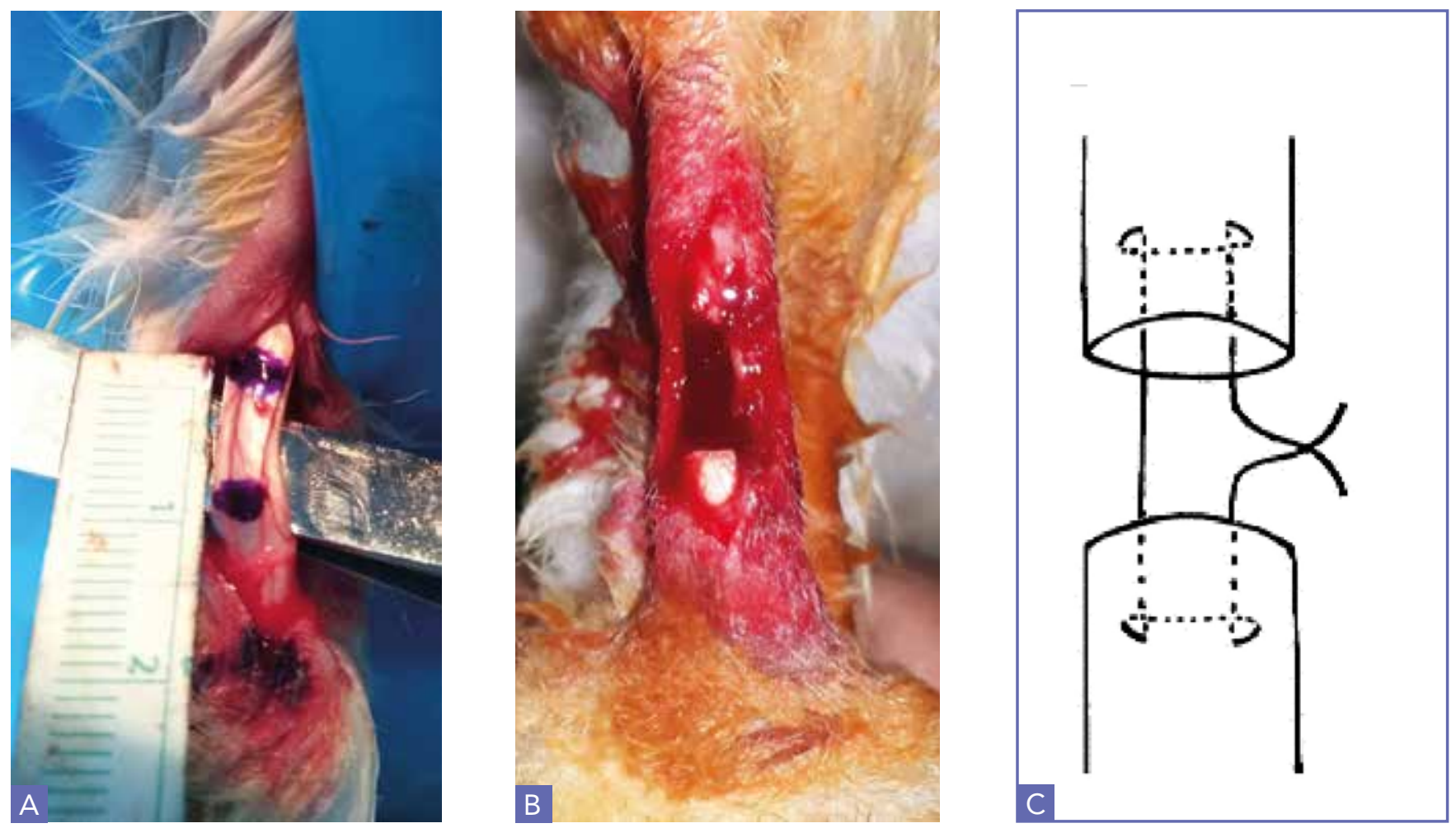

Figure 1. (a, b) Photographs showing $1 \mathrm{~cm}$ tendon defect formation in the rabbit Achilles tendon. (c) Modified Kessler Suture Method.

Group 1 (Sham): the $1 \mathrm{~cm}$ tendon defect was "splinted" leaving a $1 \mathrm{~cm}$ gap without tendon ends getting closer using non-absorbable Prolene ${ }^{\circledR}$ (3/0, polypropylene) suture, while the hind soles were positioned parallel to the ground (figure 2 a).

Group $2(\mathrm{EGF}+)$ : the same as Group 1, but with the addition of an EGF injection $(25 \mu \mathrm{g} / \mathrm{kg})$ in the defect (figure $\mathbf{2} \mathbf{b}, \mathbf{c}$ ).

Group 3 (Scaffold + EGF): the same as Group 1, but with the addition of a biodegradable, porous scaffold loaded with $25 \mu \mathrm{g} / \mathrm{kg}$ EGF (figure $2 \mathbf{d}$ ).

Postoperative $25 \mu \mathrm{g} / \mathrm{kg}$ EGF injections were made in the defect region for 10 days every other day in Group 2 and Group 3 (figure 3). Only ankle splint was applied in Group 1 as postoperative procedure.

The wounds of all rabbits were dressed on the second post-surgical day and changed each 2-day interval for 10 days. Three rabbits died during postoperative follow-up. One rabbit died in each group, leaving 9 rabbits in each group. At eight weeks post-surgery, a bloc excision was performed on the Achilles tendons of all sacrificed rabbits from the muscle-tendon junction proximal to the bone-tendon junction where the Achilles tendon adheres to the calcaneus. Repair and regeneration of sacrificed Achilles tendons were examined macroscopically, histologically, and biomechanically. Five of the 9 tendons in each group were allocated randomized (13) for biomechanical evaluation and the remaining 4 were used for histological evaluation.

\section{Histological evaluation}

Histological evaluation was performed by two independent university histology professors using an agreed upon criteria. Initial rater agreement was $\geq 80 \%$. When disagreements existed, the raters consulted with each other to finalize their assessment.

Histological scoring was performed from healing site samples using the following criteria: $0=$ no development evidence; 1 =weak development evidence; $2=$ =moderate development evidence; $3=$ strong development evidence (13). Therefore, a score of 0 for adipocytes, for example, suggests that no adipocytes were observed the defect, while a score of 3 suggests a high adipocyte volume.

For this evaluation, $1 \mathrm{~cm}$ long tendon healing area sections and an equal length from the healthy contralateral Achilles tendon (control) were excised, and then rapidly fixed in $10 \%$ formalin. All tissue specimens were then processed 

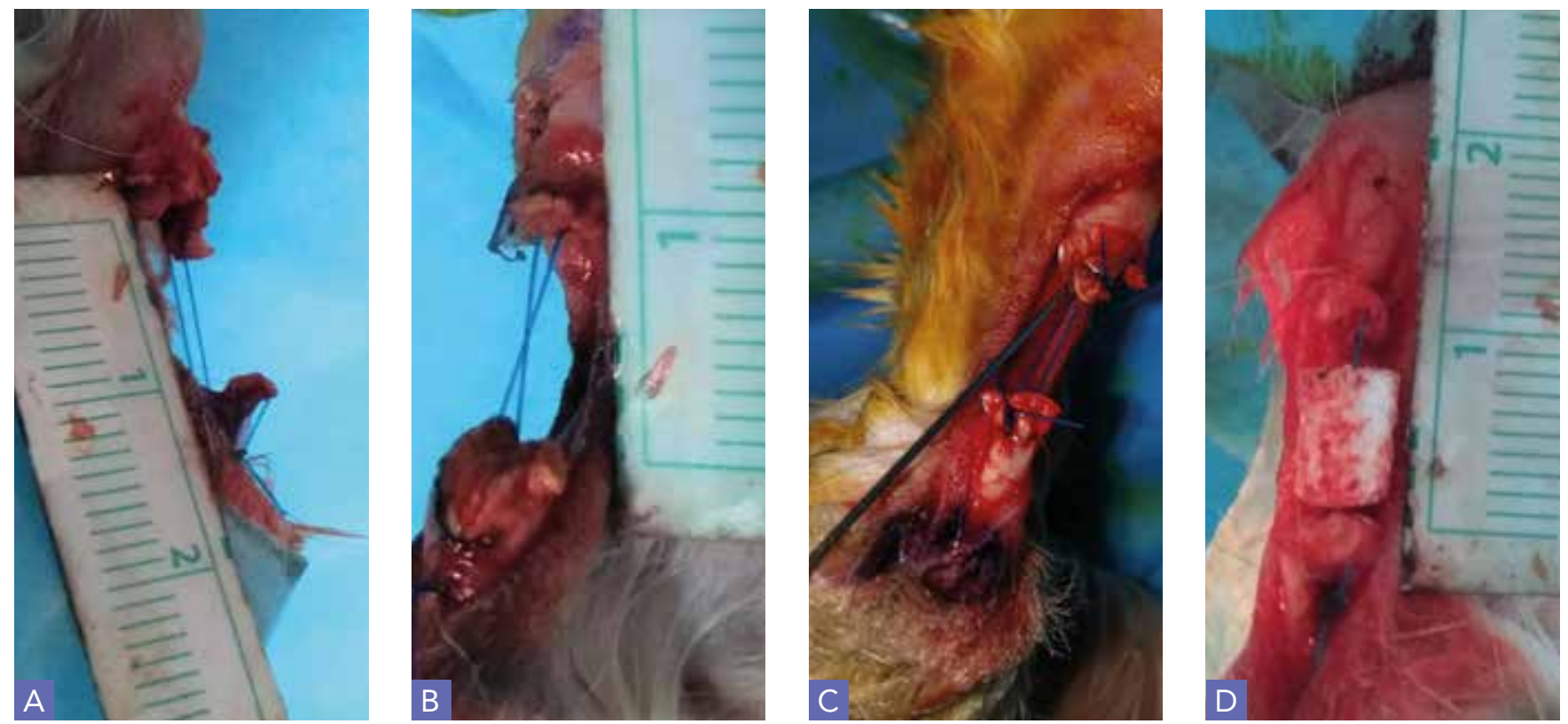

Figure 2. (a) Photograph showing surgical repair in Group 1 (Sham). (b, c) Photographs showing surgical repair and EGF injection on the Achilles tendon rupture site in Group 2 (EGF +). (d) Photograph showing surgical repair by grafting the Achilles tendon rupture defect with scaffold and impregnating the scaffold with EGF in Group 3 (Scaffold + EGF).

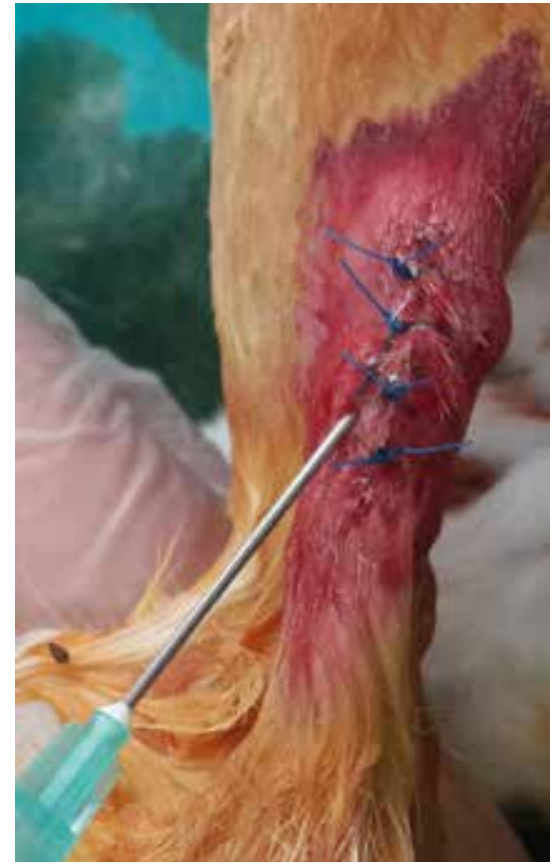

Figure 3. Photographs showing EGF injection, which were performed every other day for 10 days in Group 2 (EGF + ) and Group 3 (Scaffold + EGF). for routine light microscopy. All tissues were embedded in paraffin, and $10 \mu \mathrm{m}$ thick sections were cut using a sliding microtome and stained with hematoxylin and eosin and Masson's trichrome. Sections were imaged digitally under a research microscope. Whole section imaging was performed using with MBF Bioscience Microlucida system (Williston USA) which consists of a motorized microscope (Leica DM4000) and an Optronics Microfire digital camera. Surgical side tissue samples were investigated comparatively in all groups for collagen fibril structure and pattern, changes in vascularity, and changes in adipocyte and inflammatory cell infiltration.

\section{Biomechanical evaluation}

Tendons that underwent biomechanical evaluation were immediately brought to the laboratory after harvesting for biomechanical testing. Specimens were placed in the soft tissue clamps of the test machine (Testometric, Rochdale, England) from both sides (figure 4). Tendons were strained at $10 \mathrm{~mm} / \mathrm{min}$. Stress-strain curves were obtained and the point where the force suddenly started to drop after the peak loading force was recorded (14). Moreover, elongation at failure $(\mathrm{mm})$ and Young's modulus $\left(\mathrm{N} / \mathrm{mm}^{2}\right)$ of the tendons (stiffness) were assessed. 


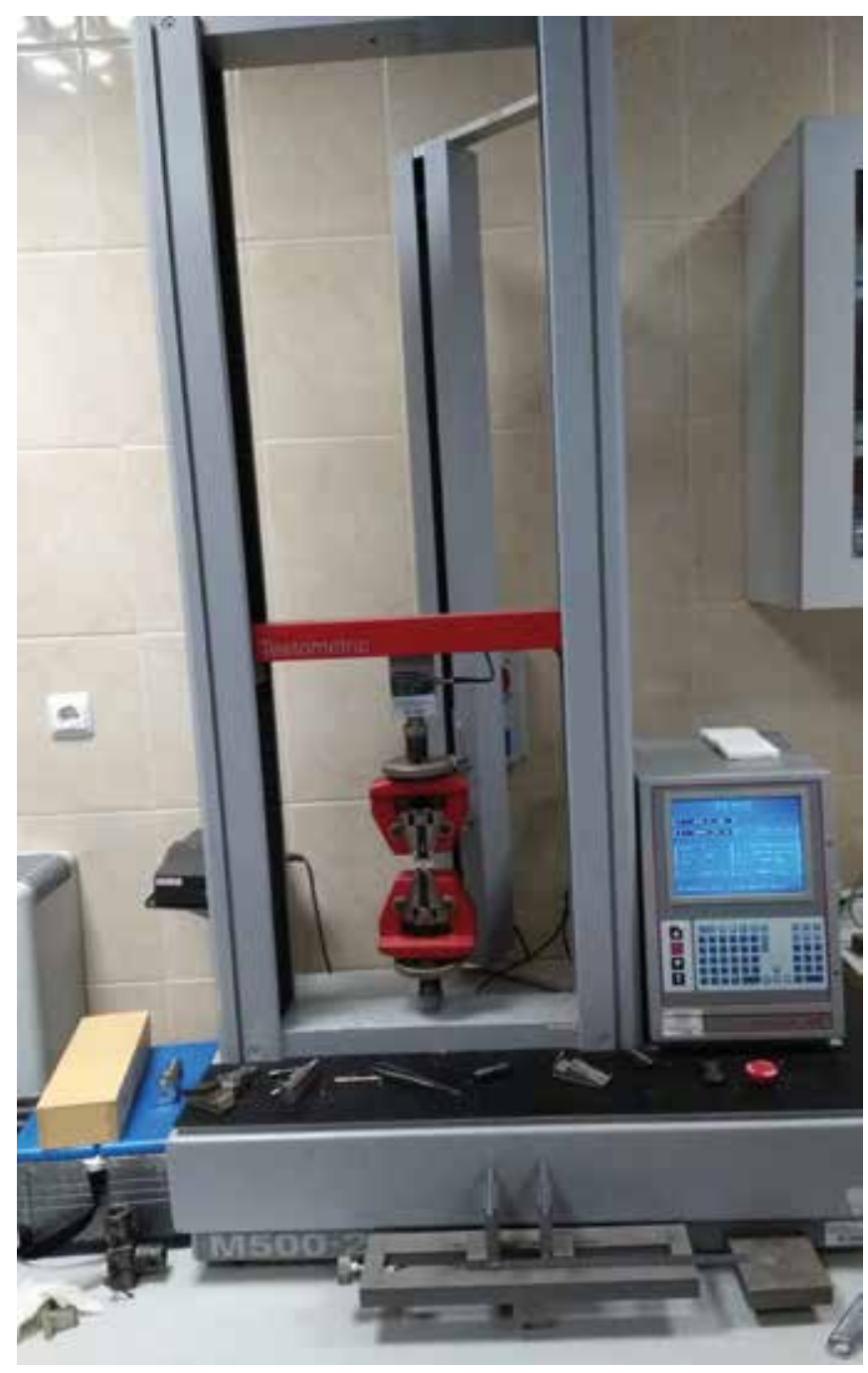

Figure 4. Biomechanical evaluation with the Testometric device.

\section{Statistical analysis}

Statistical analysis was performed to compare the values obtained from the biomechanical tendon studies. In determining whether there were statistically significant differences between the 3 experimental groups and the control group for load at failure force, Young's modulus, and elongation at failure, the Shapiro-Wilks test was performed to confirm data normality. Following this, one-way Analysis of Variance (ANOVA) was applied to each of the 3 variables. Variance homogeneity assumption was evaluated using the Levene test. Post-hoc analysis was performed in order to better delineate which group created the difference for differences between the groups identified in Young's modulus variable, and the Bonferroni test was performed for paired comparisons at this stage. Fisher's exact tests were used to delineate group differences for histological scoring variables. All analyses were performed using the SPSS Version 22.0 statistical software package (IBM-SPSS, Armonk, NY, USA). An alpha level of $\mathrm{p}$ $<0.05$ was selected to indicate statistical significance.

\section{RESULTS}

\section{Macroscopic findings}

Defect healing and bridging was macroscopically observed in all subject groups (figure 5 a-c).

\section{Histological findings}

Group 1 (Sham): new vessel formation, i.e. angiogenesis and Type III collagen were observed to be newly constructed tendon tissue. Rare adiposity was observed (figure 6).

New vessel formation was observed in the defect area, which was thought to originate from the pericytes adjacent to the endothelial cells present along the defect line. Therefore, growth factors and cells necessary for healing appeared in the defect area. Adipocytes and pericytes were also observed in association with new vessel formation.

Group 2 (EGF +): under light microscopy, tendon angiogenesis was observed. Group 2 displayed greater evidence of vascularization (moderate) than Group 1 and Group 3, $(\mathrm{p}=0.006)$. It was also observed that pericytes accumulated along the defect and healing continued. The concentration of adipocytes was greater than in Group 1. Group 2 displayed greater evidence of adipocyte development (moderate) than Group 1 and Group 3 ( $\mathrm{p}<0.0001$ ) (figure 7). Increased adipocyte concentration suggests that tendon repair was more active in accelerated in Group 2 compared with Group 1 and Group 3. Group 2 displayed greater evidence of Collagen Type III development (moderate) than Group 1 and Group 3 ( $\mathrm{p}=0.006$ ). In contrast to Group 1, Group 2 also exhibited small quantities of Type I collagen fibers. The fact that Type I collagen was observed in this group, even in small quantities at 8 weeks post-repair indicates that tendon healing was at a more advanced stage and the healing progression was advanced compared with Group 1. Group 2 and Group 3 displayed greater evidence of peripheral nerve development (weak) than Group $1(\mathrm{p}=0.006)$.

Peripheral nerve buds that we considered differentiated from MSCs through the injection of EGF were also observed in Group 2 but not in Group 1 (figure 7).

Group 3 (Scaffold + EGF): under light microscopy, tendon angiogenesis was observed. It was also observed that pericytes with vascularization accumulated along the defect and healing continued. Vessel formation in the tendon, i.e. 

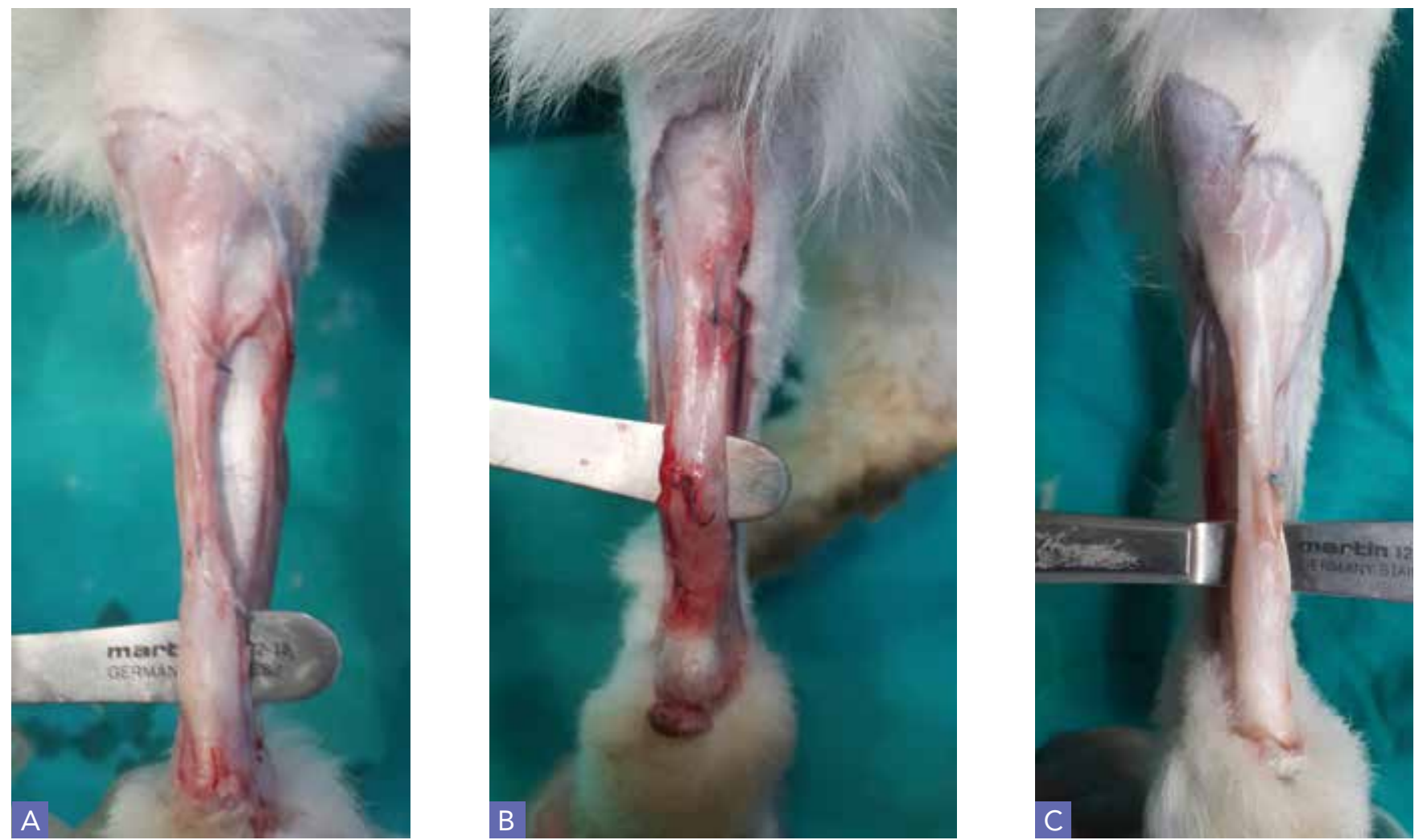

Figure 5. (a) Group 1 (Sham), (b) Group 2 (EGF +) and (c) Group 3 (Scaffold + EGF).

angiogenesis was observed. Peripheral nerve buds that were considered to have differentiated from mesenchymal stem cells were also observed in this group.

Group 3 displayed greater evidence of Collagen Type I development (moderate) than Group 1 and Group 2 ( $\mathrm{p}<0.0001$ ) (table I), suggesting more accelerated healing as Type III collagen was replaced by Type I collagen. The amount of Type III collagen was lower and Type I collagen was higher in Group 3 even compared to Group 2 (table I). The level of Achilles tendon healing that was observed in this group more closely resembled normal tendon structure. We perceive that with combined scaffold and EGF application, the necessity to produce Type III collagen to serve as a temporary healing scaffold was diminished so tissue plasticity was biased more toward Type I collagen transformation compared to the other experimental groups (figure 8). The amount of adipocytes was lower compared with Group 2 since this group was at a last phase of healing process where type I collagen making process is mostly accomplished (table I). This group, which was dominated by Type I collagen, appearing to have moved past the active proliferation phase by 8 weeks following defect repair, with lesser energy needs during the final phase of healing.

\section{Biomechanical test findings}

Peak load to failure forces were identified as $325.8 \pm 101.7$ $\mathrm{N}$ in the control group, $415.7 \pm 111.5 \mathrm{~N}$ in Group 1, 314.7 $\pm 72 \mathrm{~N}$ in Group 2, and 311.1 $\pm 94.9 \mathrm{~N}$ in Group 3 group (table II). When Group 1, Group 2, and Group 3 were compared with each other and with the control group, significant group differences were not evident $(p>0.05)$ (figure 9).

The amount of elongation at failure $(\mathrm{mm})$ was identified as $7.4 \pm 2 \mathrm{~mm}$ in the control group, $10.4 \pm 4.3 \mathrm{~mm}$ in Group 1, $12 \pm 5 \mathrm{~mm}$ in Group 2 and $11.4 \pm 5.3 \mathrm{~mm}$ in Group 3 (table II). There were no significant statistical differences between the groups $(\mathrm{p}>0.05)$.

Among the three different biomechanical characteristics that were evaluated, a significant statistical difference was only identified for Young's modulus (stiffness) values ( $\mathrm{p}$ $<0.05$ ). Pairwise comparisons revealed that Group 2 and Group 3 displayed less stiffness compared to the control group $(\mathrm{p}<0.05)$. Pairwise comparison between Group 1, Group 2 and Group 3 did not reveal statistically significant Young's modulus differences $(\mathrm{p}>0.05)$ (figure 10). 
I. A. KoCYIGIT, G. HURI, S. YÜRÜKER, ET AL.
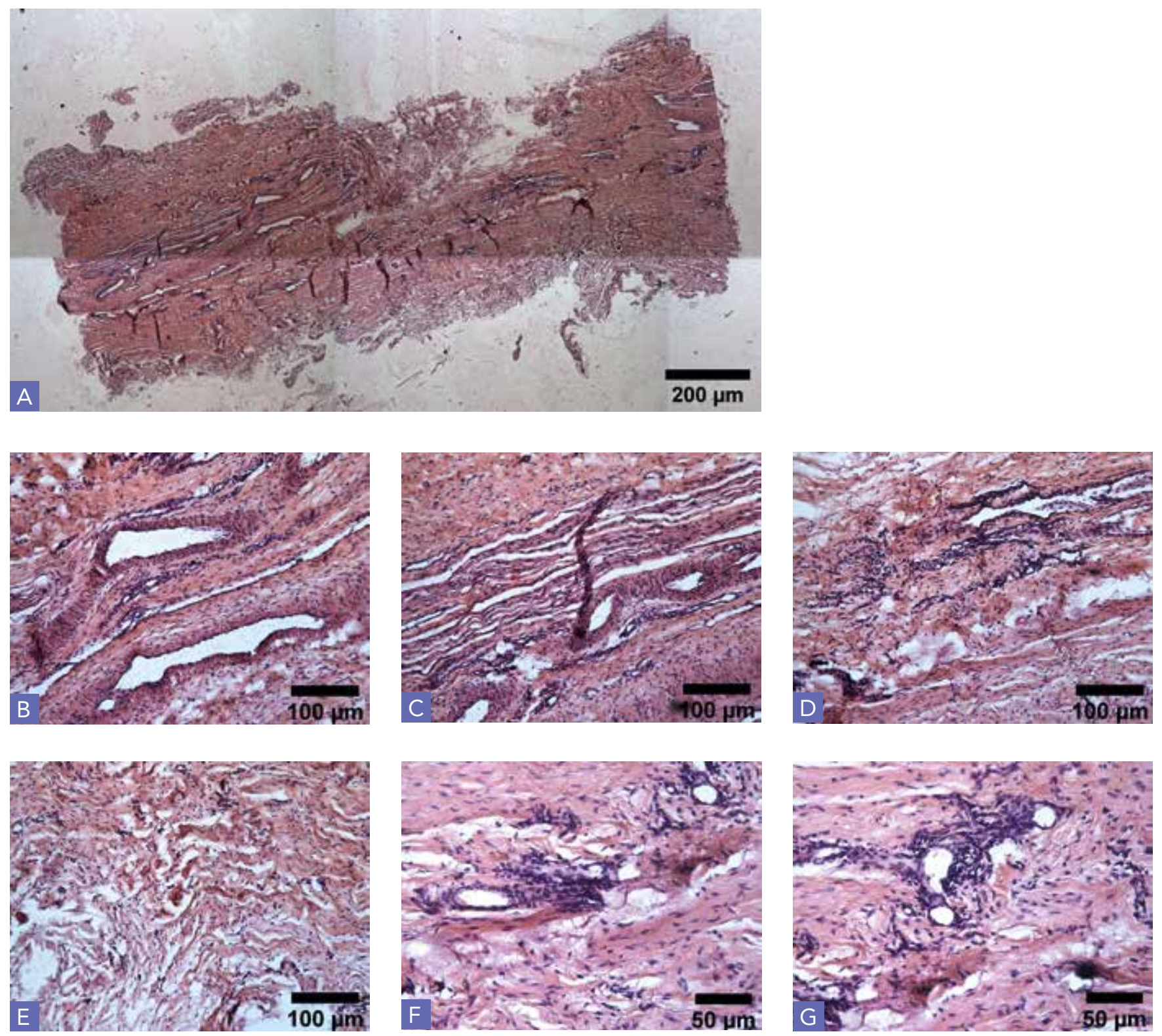

Figure 6. Histologic evaluation of tendon regeneration in Group 1 (Sham).

Table I. Histological group comparisons.

\begin{tabular}{lccc}
\hline & SHAM & EGF & EGF + SCAFFOLD \\
\hline Collagen type III & 1 & 2 & 1 \\
\hline Collagen type I & 0 & 1 & 2 \\
\hline Adipocyte & 0 & 2 & 1 \\
\hline Vascularization & 1 & 1 & 1 \\
\hline Peripheral nerve & 0 & 1 & 1 \\
\hline
\end{tabular}



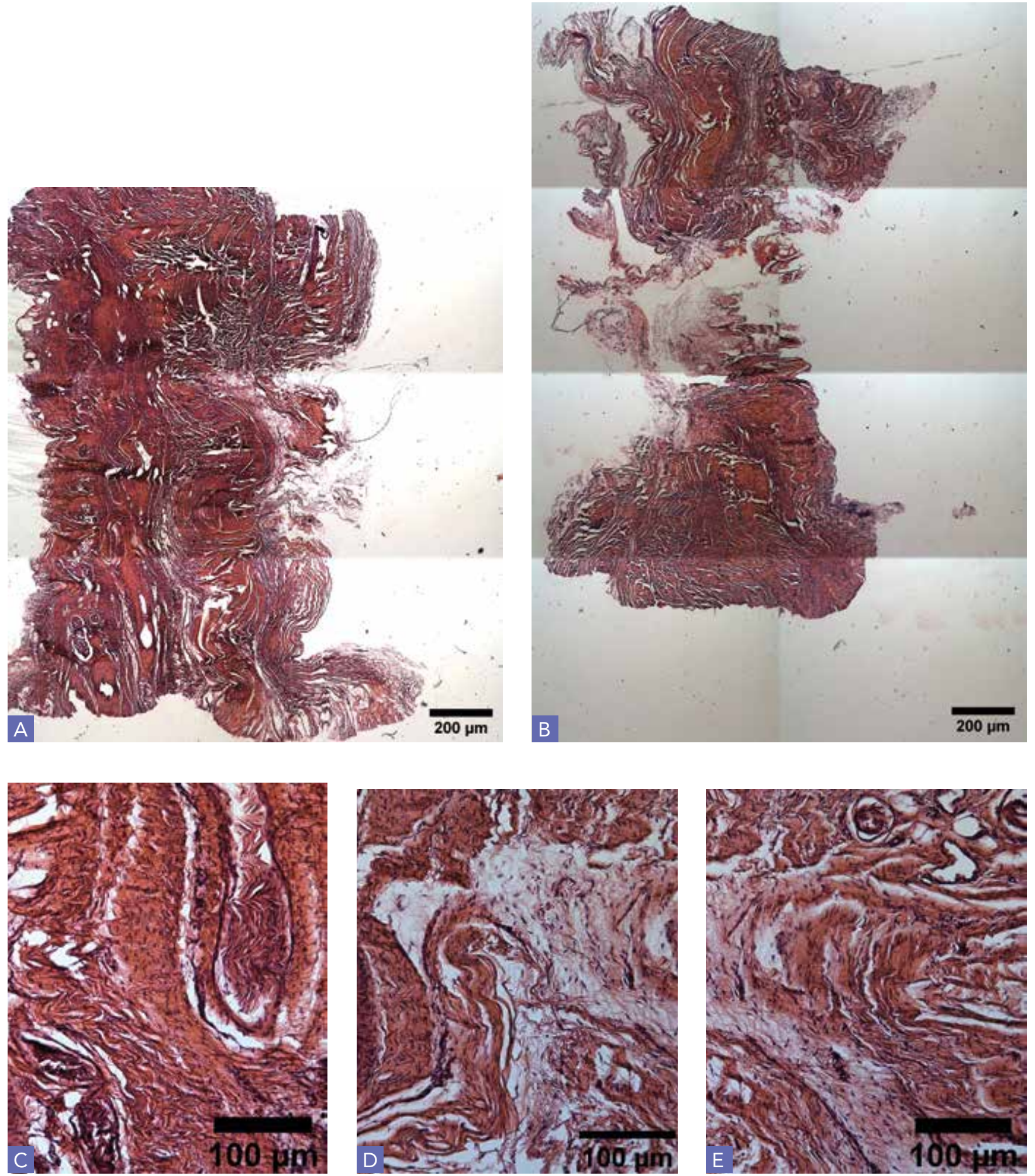

Figure 7. Type III > Type I collagen dominance and peripheral nerve buds were observed. Adipocytes were also observed. Group $2(\mathrm{EGF}+)$. 

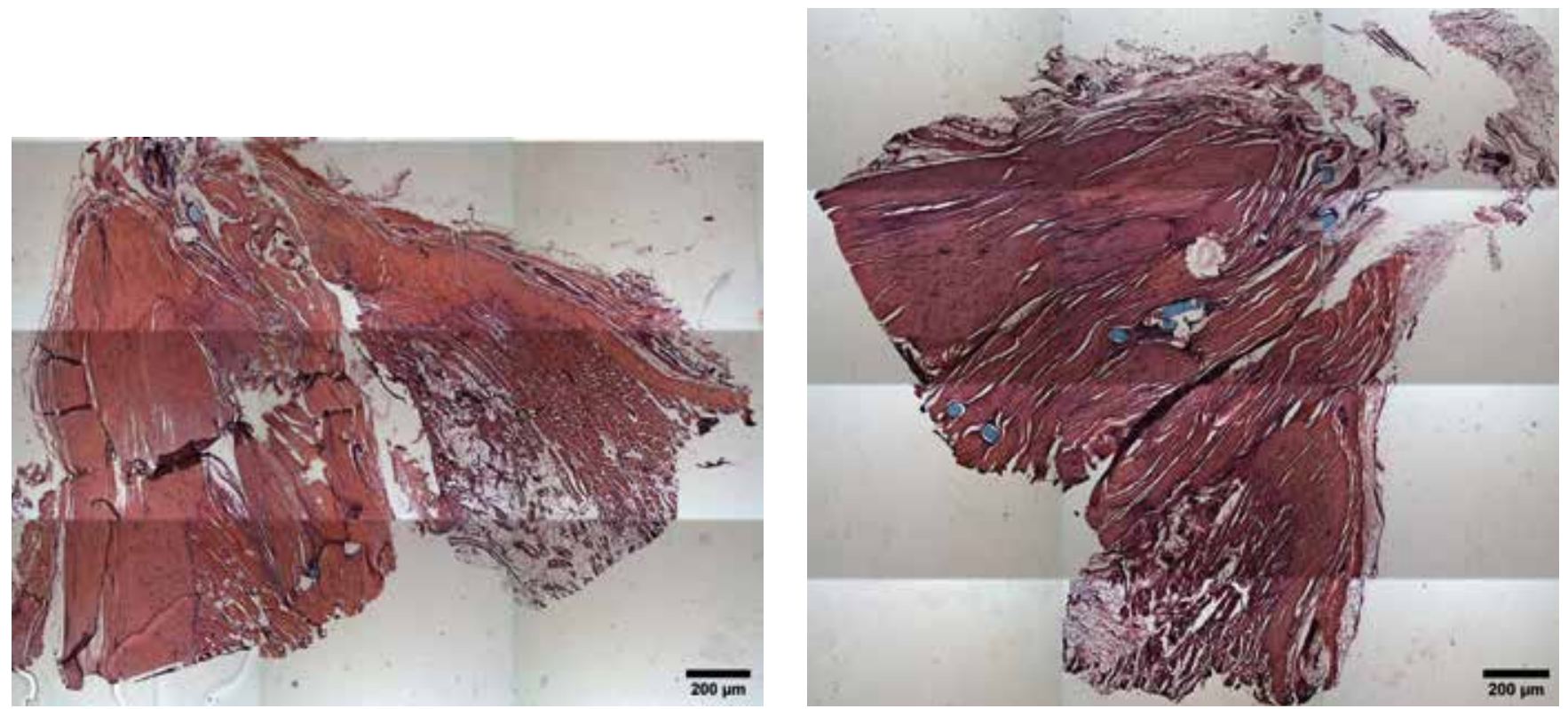

Figure 8. Type I collagen dominance was observed in Group 3 (Scaffold + EGF).

Table II. Biomechanical test group comparisons.

\begin{tabular}{|c|c|c|c|c|}
\hline & & $\mathbf{N}$ & Mean & Std. Deviation \\
\hline \multirow[t]{4}{*}{ Force Peak $(\mathrm{N})$} & Control & 5 & 325.8 & 101.7 \\
\hline & Group 1 (Sham) & 5 & 415.8 & 111.6 \\
\hline & Group 3 (Scaffold + EGF) & 5 & 311.1 & 95.0 \\
\hline & Total & 20 & 341.8 & 98.7 \\
\hline \multirow{3}{*}{ Elongation at Failure $(\mathrm{mm})$} & Group $2(\mathrm{EGF}+)$ & 5 & 12.1 & 5.0 \\
\hline & Group 3 (Scaffold + EGF) & 5 & 11.4 & 5.3 \\
\hline & Total & 20 & 10.4 & 4.4 \\
\hline \multirow[t]{2}{*}{ Young modulus $\left(\mathrm{N} / \mathrm{mm}^{2}\right)$} & Control & 5 & 190.6 & 61.4 \\
\hline & Total & 20 & 124.8 & 57.9 \\
\hline
\end{tabular}

\section{DISCUSSION}

Current approaches to Achilles tendon rupture treatment involve surgical repair and early mobilization (15). The goal of surgical repair is to minimize the risk of tendon re-rupture and prolonged immobilization by creating a repair that has sufficient fixation strength, without negatively affecting natural tendon physiology (15-17). The more closely the healing tendon maintains natural anatomical and histological characteristics the lower the re-rupture and complication rates should be while still enabling earlier mobilization or therapeutic exercise performance.

There was no significant difference in strength between Krackow, Bunnell and Kessler suture techniques in Achilles tendon repairs in a human cadaver study (18). Karatekin et 


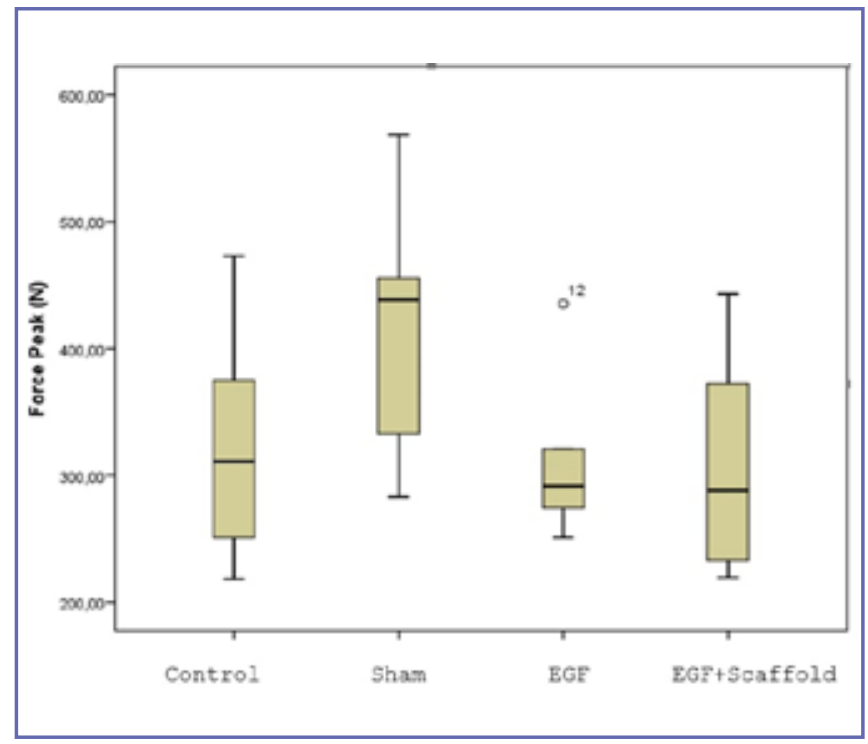

Figure 9. Peak load to failure.

al. reported similar functional and elastographic results of Krackow and modified Kessler suture methods in the longterm follow-up of the patients (19). Modified triple Kessler repair was stronger in this cadaveric biomechanical study compared with the traditionally used single Krackow technique. The modified Kessler technique was used because it was thought to be superior or not biomechanically different compared to other suture techniques.

Unfortunately, Achilles tendon healing following surgical repair often results in a fibrovascular scar that is mechanically weaker than the native tendon. This scar formation occurs in association with Type I/Type III collagen ratio changes, with greater Type III collagen fiber concentrations. Tendon healing strategies aim to bring this collagen type ratio as close as possible to that of healthy tendon (20). When applied within the initial 10 days following tendon rupture, growth factors accelerate tendon healing and reduce adhesions $(7,8)$. When growth factors and other biologic healing agents are concentrated in a given medium, the healing process can become accelerated (8).

Various biologic agents and growth factors are currently used in combination with surgical interventions $(9,21)$. However, there is no consensus or guidelines concerning the use of biologic agents and growth factors for Achilles tendon repair. Platelet Rich Plasma (PRP) releases various growth factors (e.g. TGF- $\beta$, PDGF, IGF, and VEGF) to enhance healing through accelerated revascularization $(20,22)$ However, a rabbit model study revealed that PRP injections led to decreased collagen fiber diameter (23). The use of PRP was also reported to be insufficient in the treat-

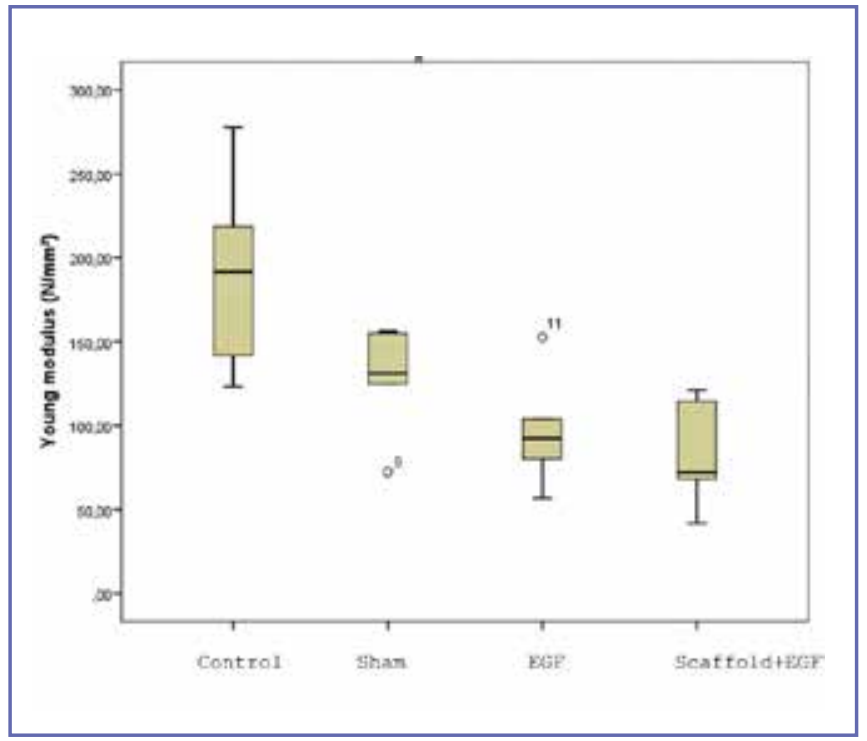

Figure 10. Young's modulus (construct stiffness).

ment of chronic Achilles tendinopathies (24-26). Additionally, PRP has not been shown to be better than placebo for human Achilles tendon repair $(6,27,28)$.

Bone marrow aspirate is a biologic agent that releases MSC to the injury site. In a pre-clinical Achilles tendon rupture study Schepull et al. (29) reported that bone marrow aspirate applied to healing tendons provided poorer outcomes compared to tendons that received MSC and a non-treated control group. Achilles tendon repair can be improved by MSC use to increase the anti-apoptotic effect (30). Using a rabbit model study, Selek et al. (31) reported that MSC use increased collagen organization over the initial 3 weeks of healing, however, significant differences were not observed during the later healing periods. Although there have been major study findings indicating that MSC use enhances tendon repair, Kraus et al. (32) reported that rat Achilles tendon repairs supplemented with MSC use displayed poorer biomechanical test results than a control group at 28 days post-injury (32).

Various growth factors are also used in tendon rupture treatments. Increased FGF levels during the early healing phase display angiogenic and mitogenic effects, and increased Type I and Type III collagen fiber production. Fibroblast growth factor and platelet-derived growth factor were shown to stimulate proliferation of mature tendon fibroblasts in serum-free medium (33). Use of FGF has been reported to have a positive effect on rat rotator cuff rupture healing (34). Use of rhPDGF can accelerate tendon healing through several mechanisms. Small animal studies have shown that rhPDGF use enhances tendon mechani- 
cal strength and movement range through matrix remodeling, collagen synthesis, improved blood clot formation and cell proliferation $(35,36)$. Use of TGF- $\beta$ has also been shown to regulate cell differentiation and proliferation, and increase Type I and Type III collagen fiber production (37). Hou et al. (38) demonstrated that tendons treated with TGF- $\beta$ displayed an increased resistance to mechanical forces (38). Animal model studies using IGF to induce tenocyte migration and collagen synthesis are ongoing. Use of VEGF increases angiogenesis and capillary permeability (39). Zhang et al. (37) reported that although there was a significant increase in Achilles tendon repair tensile strength at two weeks following VEGF application, there was no significant differences by the end of the $4^{\text {th }}$ week (40). Each of the above-mentioned biological agents show varying levels of promise for Achilles tendon repair, however, only a few techniques have achieved clinical application. Therefore, at the current time there is no biologic agent available that constitutes a standard treatment protocol for Achilles tendon rupture with a consensus that healing will be obtained that more closely resembles that of native, healthy tendon tissue.

Although healthy tendons are dominated by Type I collagen fibers, a significant amount of Type III collagen fibers get synthesized in the repair site following rupture and repair which gradually transform into Type I collagen fibers during the later healing stages. Type III collagen has a very low resistance to tensile forces, therefore, the healing tendon is more susceptible to spontaneous rupture during this time period (41). The aim in tendon repair treatment is to achieve Type I collagen dominance by the end of the healing process. The higher the Type I/Type III ratio the lower the re-rupture and complication rate (42). Conceivably, earlier Type III to Type I collagen fiber transformation should help prevent re-rupture or repair site stretching during recovery while enabling earlier mobilization.

To date, to our knowledge, no pre-clinical or clinical studies regarding EGF use for tendon healing have been reported. Local EGF use has been clinically shown to improve chronic skin wound healing, closure, and epidermal bridging (11). Using a rat model, Brown et al. (43) reported increased collagen formation following EGF application at surgical incision sites, reporting a $200 \%$ increase in wound tensile strength at 7-14 days post-application. Clinical studies of EGF embedded in silver sulfadiazine has been reported to increase the epithelialization of chronic wounds, particularly when applied using intra- and peri-lesional injections (10). Various biologic agents have been used to facilitate tendon healing. Debates exists as to which agent(s) should be used and at what time they should be delivered during the healing process. To improve this understanding, we used a $1 \mathrm{~cm}$ defect Achilles tendon defect model to evaluate the efficacy of EGF applications over the initial 10 days post-injury. Histologic evaluation revealed that within the same 8-week time period Group 1 displayed less Type III collagen fiber formation than Group 2. Group 2 displayed significantly more Type III collagen fiber formation in combination with some evidence of earlier transformation to Type I collagen fibers. Therefore, the addition of EGF was found to accelerate Type III to Type I collagen fiber synthesis. Group 2 tendon healing was noted to be more advanced compared to Group 1.

Histologic examination revealed that Group 3 had the greatest level of Type I collagen fibers. Group 3 also had fewer adipocytes and Type III collagen fibers than Group 2. These findings suggest that Group 3 had moved past the active proliferation healing phase over the same period and the need for adipocytes decreased in proportion to the reduced active synthesis. The accelerated transformation of Type III to Type I collagen fibers was significantly more evident in this group. With the addition of a PCL scaffold of sufficient size to fill the defect, all tendon healing phases took place more quickly and more robustly. These positive findings using a PCL scaffold are in agreement with over reports (44).

Scaffolds are known to provide both mechanical support and guidance for migrating cells to grow during tendon healing (45). This study used biodegradable scaffolds made of PCL which is known to facilitate bone defect repair based on an animal model study (46). An optimal Achilles tendon repair scaffold should enable more natural healing and rapid tendon defect bridging (44). The scaffolds produced can be processed in a perfusion reactor, cultivating tenocytes or tendon precursor stem cells, to verify and to optimize the interaction between the tissue engineered structure and the cells (47). Growth factors are susceptible to degradation and their efficacy can be quickly reduced due to rapid elimination from the defect site following injection. When growth factors are encapsulated within scaffolds, they are maintained for a comparatively longer time period. Therefore, better tendon healing can be achieved by using scaffold and growth factors in combination. To sum up the histological findings, the addition of EGF accelerated healing, whereas scaffold and EGF use in combination led to the quickest histologic healing. Moreover, an unexpected outcome of this study was the peripheral nerve buds that were observed in the tendon following EGF injections.

Biomechanical testing revealed that load at failure and elongation at failure values revealed no differences between the 3 experimental groups. The only significant biomechanical characteristic difference was for Young's modulus (stiffness). Both Group 2 and Group 3 displayed lower Young's modu- 
lus values compared to the control group (healthy contralateral Achilles tendon). Biomechanically, the difference between the groups showed that the addition of EGF and scaffold did not have a positive biomechanical contribution. Achilles tendon rupture treatment with lentiviral bFGF transduced mesenchymal stem cells did not show positive effects biomechanically in a long-term follow-up. Interestingly, in later stages stem cells had hardly any effects on biomechanical results (48). The PRGF did not have a major influence on cellular organization. It also had an undesirable effect on the biomechanical properties of repaired flexor tendons (49). In another PRGF study, the PRGF-treated tendons had higher force at 8 weeks compared with the placebo group biomechanically (50). Histologically, immediate injection of PRP for tendon injury improves tendon healing in rats. Nevertheless, PRP injection group in Achilles tendon injury had lower peak force biomechanically than the control group (51). Zhang et al. reported administration of exogenous VEGF can significantly improve tensile strength early in the course of the rat Achilles tendon healing but there was no significant difference in tensile strength among the groups at 4 weeks postoperatively (39). Application of the TGF-B1 and IGF-1 in a patellar tendon defect model resulted in a significant increase in force at failure, ultimate stress, stiffness, and energy uptake at 2 weeks, whereas none of the parameters revealed any significant difference between the two groups at 6 weeks (52). Local application of FGF-2 on tendon-to-bone remodeling in rats was reported FGF-treated specimens were stronger at 2 weeks but at 4 and 6 weeks, both specimens were exhibited similar strength biomechanically (53). In studies that added growth factors to tendon repair, there was no significant difference in long-term results with the control group, as in our study.

In terms of clinical practice, tendon biomechanics study is more important than histological study. Since there is no biomechanical positive effect with the addition of EGF and

\section{REFERENCES}

1. Nunley JA. The Achilles Tendon: Treatment and Rehabilitation. New York: Springer, 2008:pp. 209-214.

2. Williams J. Achilles tendon lesions in sport. Sports Med 1993;16(3):216-20.

3. Kannus P, Natri A. Etiology and pathophysiology of tendon ruptures in sports. Scand J Med Sci Sports 1997;7(2):107-12.

4. Doral MN. What is the effect of the early weight-bearing mobilisation without using any support after endoscopy-assisted Achilles tendon repair? Knee Surg Sports Traumatol Arthrosc 2013;21(6):1378-84.

5. Doral MN, Bozkurt M, Turhan E, et al. Achilles tendon rupture: physiotherapy and endoscopy-assisted surgical treat- scaffold, it is not expected to reduce early joint movement start time, work turnaround time and risk of re-rupture. Although the addition of EGF is histologically significant, more studies are needed to use it in daily clinical practice.

\section{Study limitations}

This study is limited in that it represents the histological and biomechanical results of a small sample of rabbit Achilles tendons at only the $8^{\text {th }}$ week post-injury. There are no midor long-term results. Additional studies are needed to determine if significant histological and biomechanical group differences exist at shorter or longer durations than 8 weeks. It may have affected the results since sufficient postoperative time has not been applied to the splint.

\section{CONCLUSIONS}

The combined addition of EGF and a scaffold to Achilles tendon defect treatment increased the Type I/Type III collagen ratio and provided better histological evidence for tendon healing compared to EGF alone or Sham conditions. Histological evaluation revealed that EGF use increased vascularization, pericyte concentration adjacent to vessel endothelial cells and adipocyte concentrations leading to accelerated and more robust tendon repair healing. There was no significant difference in terms of biomechanics with the addition of EGF and scaffold. Although addition of EGF is histologically significant, it has no advantage over control and sham groups biomechanically. Therefore, further studies with larger sample sizes and over longer study durations are needed prior to clinical use of EGF for tendon rupture treatment.

\section{CONFLICT OF INTERESTS}

The authors declare that they have no conflict of interests.

ment of a common sports injury. Open access J Sports Med 2010;1:233

6. de Jonge S, de Vos RJ, Weir A, et al. One-year follow-up of platelet-rich plasma treatment in chronic achilles tendinopathy a double-blind randomized placebo-controlled trial. AmJ Sports Med 2011;39(8):1623-9.

7. Tsubone T, Moran SL, Amadio PC, Zhao C, An KN. Expression of growth factors in canine flexor tendon after laceration in vivo. Ann Plast Surg 2004;53(4):393-7.

8. Shapiro E, Grande D, Drakos M. Biologics in Achilles tendon healing and repair: a review. Curr Rev Musculoskelet Med 2015;8(1):9-17. 
9. Oliva F, Gatti S, Porcellini G, Forsyth NR, Maffulli N. Growth factors and tendon healing. Med Sport Sci 2012;57:53-64.

10. Currie LJ, Sharpe JR, Martin R. The use of fibrin glue in skin grafts and tissue-engineered skin replacements. Plast Reconstr Surg 2001;108:1713-26.

11. Leonida MD, Kumar I. Bionanomaterials for Skin Regeneration. Springer :pp. 27-35.

12. Z Zorlutuna P, Tezcaner A, Hasirci V. A novel construct as a cell carrier for tissue engineering. J Biomater Sci Polym Ed 2008;19(3):399-410.

13. Padulo J, Oliva F, Frizziero A, Maffulli N. Muscles, Ligaments and Tendons Journal - Basic principles and recommendations in clinical and field Science Research: 2018 update. MLTJ 2018;8(3):305-307.

14. Maffulli N, Longo UG, Franceschi F, Rabitti C, Denaro V. Movin and Bonar scores assess the same characteristics of tendon histology. Clin Orthop Relat Res 2008;466(7):1605-11.

15. Yilmaz G, Doral MN, Turhan E, et al. Surgical treatment of Achilles tendon ruptures: The comparison of open and percutaneous methods in a rabbit model. Ulus Travma Acil Cerrahi Derg 2014;20(5):311-8.

16. Maffulli N, Tallon C, Wong J, Lim KP, Bleakney R. Early weightbearing and ankle mobilization after open repair of acute midsubstance tears of the achilles tendon. Am J Sports Med 2003;31(5):692-700.

17. Speck M, Klaue K. Early full weightbearing and functional treatment after surgical repair of acute achilles tendon rupture. Am J Sports Med 1998;26(6):789-93

18. Suchak AA, Spooner C, Reid DC, Jomha NM. Postoperative rehabilitation protocols for Achilles tendon ruptures: a meta-analysis. Clin Orthop Relat Res 2006;445:216-21.

19. McCoy BW, Haddad SL. The strength of achilles tendon repair: a comparison of three suture techniques in human cadaver tendons. Foot Ankle Int 2010;31(8):701-5.

20. Karatekin YS, Karaismailoglu B, Kaynak G, et al. Does elasticity of Achilles tendon change after suture applications? Evaluation of repair area by acoustic radiation force impulse elastography. J orthop Surg Res 2018;13(1):1-7.

21. Magnusson S, Qvortrup K, Larsen JO, et al. Collagen fibril size and crimp morphology in ruptured and intact Achilles tendons. Matrix biol 2002;21(4):369-77.

22. Ohba S, Hojo H, Chung UI. Bioactive factors for tissue regeneration: state of the art. MLTJ 2012;2(3):193.

23. Guevara-Alvarez A, Schmitt A, Russell RP, Imhoff AB, Buchmann S. Growth factor delivery vehicles for tendon injuries: Mesenchymal stem cells and Platelet Rich Plasma. MLTJ 2014;4(3):378.

24. Soomekh DJ. Current concepts for the use of platelet-rich plasma in the foot and ankle. Clin Podiatr Med Surg 2011;28(1):155-70.

25. Dallaudière B, Lempicki M, Pesquer L, et al. Efficacy of intra-tendinous injection of platelet-rich plasma in treating tendinosis: comprehensive assessment of a rat model. Eur radiol 2013;23(10):2830-7.

26. Vannini F, Di Matteo B, Filardo G, Kon E, Marcacci M, Giannini S. Platelet-rich plasma for foot and ankle pathologies: a systematic review. Foot Ankle Surg 2014;20(1):2-9.

27. Sarrafian TL, Wang H, Hackett ES, et al. Comparison of Achilles tendon repair techniques in a sheep model using a cross-linked acellular porcine dermal patch and platelet-rich plasma fibrin matrix for augmentation. J Foot Ankle Surg 2010;49(2):128-34.

28. Moraes VY, Lenza M, Tamaoki MJ, Faloppa F, Belloti JC. Platelet-rich therapies for musculoskeletal soft tissue injuries. The Cochrane Library 2014.

29. Solchaga LA, Bendele A, Shah V, et al. Comparison of the effect of intra-tendon applications of recombinant human platelet-derived growth factor-BB, platelet-rich plasma, steroids in a rat achilles tendon collagenase model. J Orthop Res 2014;32(1):145-50.

30. Schepull T, Kvist J, Norrman H, Trinks M, Berlin G, Aspenberg P. Autologous platelets have no effect on the healing of human achilles tendon ruptures: a randomized single-blind study. Am J Sports Med 2011;39(1):38-47.

31. Okamoto N, Kushida T, Oe K, Umeda M, Ikehara S, Iida $\mathrm{H}$. Treating Achilles tendon rupture in rats with bone-marrow-cell transplantation therapy. J Bone Joint Surg Am 2010;92(17):2776-84

32. Selek O, Buluc L, Muezzinoğlu B, Ergün R, Ayhan S, Karaöz E. Mesenchymal stem cell application improves tendon healing via anti-apoptotic effect (Animal study). Acta Orthop Traumatol Turc 2013;48(2):187-95.

33. Kraus T, Imhoff F, Wexel G, et al. Stem Cells and Basic Fibroblast Growth Factor Failed to Improve Tendon Healing. J Bone Jt Surg 2014;96(9):761-9.

34. Stein LE. Effects of serum, fibroblast growth factor, and platelet-derived growth factor on explants of rat tail tendon: a morphological study. Cells Tissues Organs 1985;123(4):247-52.

35. Ide J, Kikukawa K, Hirose J, Iyama K-i, Sakamoto H, Mizuta H. The effects of fibroblast growth factor- 2 on rotator cuff reconstruction with acellular dermal matrix grafts. Arthroscopy. The Journal of Arthroscopic \& Related Surgery 2009;25(6):608-16.

36. Shah V, Bendele A, Dines JS, et al. Dose-response effect of an intra-tendon application of recombinant human platelet-derived growth factor-BB (rhPDGF-BB) in a rat Achilles tendinopathy model. J Orthop Res 2013;31(3):413-20.

37. Cummings SH, Grande DA, Hee CK, et al. Effect of recombinant human platelet-derived growth factor-BBcoated sutures on Achilles tendon healing in a rat model: A histological and biomechanical study. J Tissue Eng 2012;3(1):2041731412453577.

38. Klein MB, Yalamanchi N, Pham H, Longaker MT, Chan J. Flexor tendon healing in vitro: effects of TGF- $\beta$ on tendon cell collagen production. J Hand Surg 2002;27(4):615-20.

39. Hou Y, Mao Z, Wei X, et al. The roles of TGF-beta1 gene transfer on collagen formation during Achilles tendon healing. Biochem Biophys Res Commun 2009;383(2):235-9.

40. Zhang F, Liu H, Stile F, et al. Effect of vascular endothelial growth factor on rat Achilles tendon healing. Plastic and reconstructive surgery. 2003;112(6):1613-9.

41. Maffulli N. Current Concepts Review - Rupture of the Achilles Tendon. J Bone Joint Surg 1999;81(7):1019-36.

42. Müller SA, Todorov A, Heisterbach PE, Martin I, Majewski M. Tendon healing: an overview of physiology, biology, and pathology of tendon healing and systematic review of state of the art in tendon bioengineering. Knee Surg Sports Traumatol 2015;23(7):2097-105. 
43. Brown GL, Curtsinger L, Jurkiewicz MJ, Nahai F, Schultz G. Stimulation of healing of chronic wounds by epidermal growth factor. Plast Reconstr Surg 1991;88(2):189-94.

44. Suckow M, Hodde J, Wolter W, Hiles M. Repair of experimental Achilles tenotomy with porcine renal capsule material in a rat model. Journal of Materials Science: Materials in Medicine 2007;18(6):1105-10.

45. Webb WR, Dale TP, Lomas AJ, et al. The application of poly (3-hydroxybutyrate-co-3-hydroxyhexanoate) scaffolds for tendon repair in the rat model. Biomaterials 2013;34(28):6683-94.

46. Huri PY, Huri G, Yasar U, Ucar Y, Dikmen N, Hasirci N, et al. A biomimetic growth factor delivery strategy for enhanced regeneration of iliac crest defects. Biomed Mater 2013;8(4):045009.

47. Reverchon E, Baldino L, Cardea S, De Marco I. Biodegradable synthetic scaffolds for tendon regeneration. MLTJ 2012;2(3):181.

48. Kraus T, Imhoff F, Reinert J, et al. Stem cells and bFGF in tendon healing: Effects of lentiviral gene transfer and longterm follow-up in a rat Achilles tendon defect model. BMC musculoskeletal disord 2016;17(1):1-7.
49. Liao JC, He M, Gan AW, Chong AK. The effects of autologous platelet-rich fibrin on flexor tendon healing in a rabbit model. J Hand Surg 2017;42(11):928.e1-.e7.

50. López-Nájera D, Rubio-Zaragoza M, Sopena-Juncosa JJ, et al. Effects of plasma rich in growth factors (PRGF) on biomechanical properties of Achilles tendon repair. Knee Surg Sports Traumatol 2016;24(12):3997-4004.

51. Circi E, Akman YE, Sukur E, Bozkurt ER, Tuzuner T, Ozturkmen Y. Impact of platelet-rich plasma injection timing on healing of Achilles tendon injury in a rat model. Acta Orthop Traumatol Turc 2016;50(3):366-72.

52. Lyras DN, Kazakos K, Verettas D, Chronopoulos E, Folaranmi $\mathrm{S}$, Agrogiannis G. Effect of combined administration of transforming growth factor-b1 and insulin-like growth factor I on the mechanical properties of a patellar tendon defect model in rabbits. Acta Orthop Belg 2010;76(3):380.

53. Ide J, Kikukawa K, Hirose J, et al. The effect of a local application of fibroblast growth factor- 2 on tendon-to-bone remodeling in rats with acute injury and repair of the supraspinatus tendon. J Shoulder Elbow Surg 2009;18(3):391-8. 